\title{
Réformes éducatives : attentes et conduite du changement
}

Education reforms: Expectations and change management

Reformas educativas: esperas y conducta del cambio

\section{Mamadou Ndoye}

\section{OpenEdition}

Journals

Édition électronique

URL : https://journals.openedition.org/ries/9292

DOI : $10.4000 /$ ries.9292

ISSN : 2261-4265

Éditeur

France Education international

Édition imprimée

Date de publication : 30 avril 2020

Pagination : $45-55$

ISBN : 978-2-85420-626-5

ISSN : $1254-4590$

\section{Référence électronique}

Mamadou Ndoye, «Réformes éducatives : attentes et conduite du changement ", Revue internationale d'éducation de Sèvres [En ligne], 83 | avril 2020, mis en ligne le 17 juin 2020, consulté le 25 juin 2021. URL : http://journals.openedition.org/ries/9292 ; DOI : https://doi.org/10.4000/ries.9292 


\title{
Réformes éducatives : attentes et conduite du changement
}

\author{
Mamadou Ndoye \\ Ancien ministre de l'alphabétisation, \\ de l'enseignement de base et des langues \\ nationales du Sénégal
}

Le présent article s'inspire de l'approche praxique des réformes éducatives menées certes avant mais surtout de 1990 à nos jours dans le cadre de l'éducation pour tous (Jomtien, Dakar, Incheon). Ces réformes ont largement alimenté le dialogue sur les politiques et le partage d'expériences dans cette période. Il convient de préciser que si les expériences en question se situent sur tous les continents, elles ont été menées plus récemment en Afrique. Au regard du nombre de pays et de régions du monde concernés, ces réformes se déroulent dans une grande diversité de contextes qui fondent la spécificité de chacune. Dès lors, toute entreprise de classification et de typologie peut se heurter à des obstacles théoriques et pratiques difficiles à surmonter. Tout en n'ignorant pas ceux-ci, l'observation de certaines caractéristiques communes dominantes de ces réformes autorise à la tenter par une double entrée : les attentes et la conduite du changement, notamment celles impulsées par la mondialisation des réformes à travers les cadres successifs de l'éducation pour tous (EPT) lancés en 1990, 2000 et 2015, et les évaluations internationales (PISA, TIMSS, PASEC, SAQMEC). L'enjeu est triple. Il s'agit tout d'abord de catégoriser les attentes et les choix stratégiques qui permettent de distinguer les politiques en matière de réforme éducative. Ceci ouvre une compréhension plus approfondie de la nature et de l'impact de chacun de ces différents types de réformes afin de mieux éclairer l'analyse des options qui ont guidé la conduite du changement. Le second enjeu est d'identifier les défis majeurs auxquels s'est heurtée la conduite du changement. De tels défis, par leur ampleur et leur complexité, expliquent souvent les écarts entre les attentes de changement et les résultats des réformes. Une des implications est aussi que la résolution de ces défis crée les conditions critiques de réussite des réformes. La réflexion sur les leçons tirées ouvre alors la voie aux perspectives nouvelles de développement des réformes auxquelles s'articulent les tendances actuelles et à venir d'évolution des systèmes éducatifs.

\section{UNE TYPOLOGIE DES RÉFORMES}

Toute réforme éducative est porteuse d'attentes de changement positif et significatif. Celles-ci peuvent se décliner en espérances tels que buts et finalités ou en résultats attendus en relation avec des objectifs généraux et spécifiques. Dans tous les cas, il est espéré une meilleure performance du système que l'on réforme. La question est ici de définir la nature de la performance attendue. À cet égard, l'examen des politiques étatiques de réforme, sous l'angle des caractéristiques dominantes, révèle trois grands types d'attentes : 
- attente 1 : extension/généralisation de la scolarisation et élévation de l'obligation scolaire et du niveau général d'instruction de la population ;

- attente 2: amélioration des résultats de l'apprentissage/de la réussite scolaire et de l'impact de l'éducation sur le développement personnel et collectif ;

- attente 3 : promotion d'un nouveau type d'homme pour un nouveau projet de société/modèle de développement.

De telles attentes relèvent d'ambitions et de complexités différentes voire inégales. En effet, à l'exception de celles relevant de l'élitisme, les politiques modernes ont gradué leurs attentes. L'attente 1 préoccupe principalement lorsque le système laisse sur le bord de la route un grand nombre d'enfants et de jeunes ainsi exclus du droit à l'éducation. L'ambition d'inclure tous est alors légitime et hautement estimable. Cependant, elle relève d'une problématique de niveau inférieur, voire primaire, de développement du système éducatif : l'accès de base. Une fois la tâche d'extension accomplie pour l'essentiel, l'attente 2 devient la priorité. La problématique est d'autant plus complexe que, le plus souvent, on ne sait pas définir la qualité, a fortiori ce qu'est améliorer la qualité. Il suffit de se référer aux glissements conceptuels successifs de la qualité et de sa mesure. Encore aujourd'hui, les évaluations standardisées en cours se limitent à mesurer le « sommet de l'iceberg » quant à l'impact de l'éducation sur les apprenants. Pour ce qui est de l'attente 3, elle semble hors de portée pour certains et relèverait de l'utopie.

Schéma 1. Escalier des ambitions et des complexités

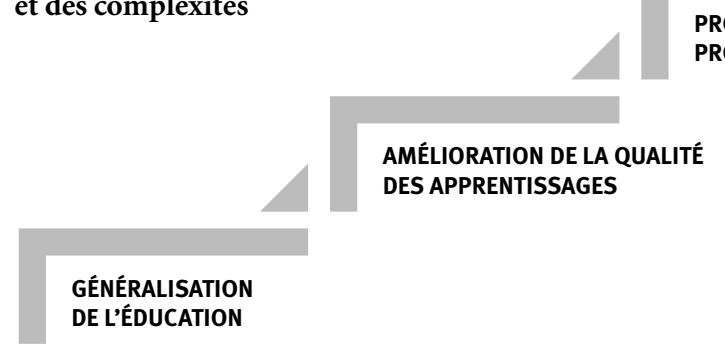

PROMOTION D'UN NOUVEAU PROJET SOCIAL

Du point de vue de la conduite du processus de réforme, la réalisation de ces attentes requiert également des choix stratégiques différents, notamment en ce qui concerne le levier principal d'action pour le changement. Les leviers principaux d'action se retrouvent dans les composantes-clés du système éducatif, décrites dans le schéma ci-dessous.

Schéma 2. Composantes clés du système

ENVIRONNEMENT SYSTÉMIQUE

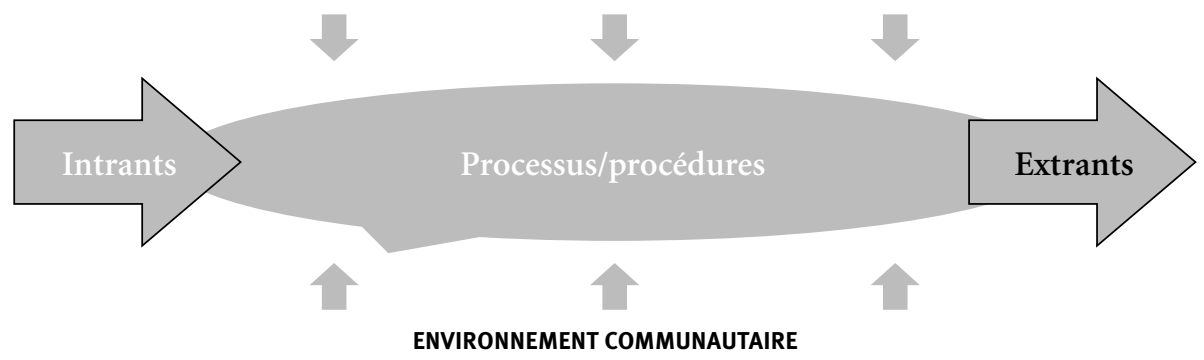


Les intrants sont d'ordre humain, matériel et intellectuel : enseignants, élèves, bâtiments scolaires, équipements, matériels d'apprentissage et curriculum. Ils sont à l'œuvre dans les processus d'enseignement/apprentissage et dans les procédures organisationnelles de l'école et de la classe qui les transforment en extrants. L'environnement systémique fournit le cadre institutionnel et administratif ainsi que l'appui financier et technique, pendant que l'environnement communautaire participe au financement, aux partenariats éducatifs et de gestion, si ces derniers lui sont ouverts. Dans la dynamique d'ensemble, chaque composante a un effet spécifique. Tout en étant en interaction avec les autres composantes, chacune possède également une relative autonomie. La notion de levier principal d'action renvoie à la composante dans laquelle le niveau d'investissement et d'impact attendu est le plus élevé pour répondre à l'attente en question. Mais il ne peut être considéré comme unique levier, en particulier eu égard aux interactions entre les composantes du système. C'est précisément l'articulation entre la caractéristique dominante de l'attente et le levier principal d'action requis pour la conduite du changement qui permet d'esquisser ainsi la typologie des réformes du point de vue de la double entrée précisée plus haut :

Tableau 1

Typologie des réformes du point de vue des attentes et du levier principal d'action

\begin{tabular}{|l|l|l|}
\hline Type de réforme & Attentes de changement & Levier principal d'action \\
\hline Additif/quantitatif & $\begin{array}{l}\text { Extension/généralisation } \\
\text { de la scolarisation }\end{array}$ & $\begin{array}{l}\text { Intrants humains } \\
\text { et matériels }\end{array}$ \\
\hline Correctif/adaptatif & $\begin{array}{l}\text { Amélioration des résultats } \\
\text { de l'apprentissage }\end{array}$ & $\begin{array}{l}\text { Intrants intellectuels, } \\
\text { processus et procédures }\end{array}$ \\
\hline $\begin{array}{l}\text { Refondateur/ } \\
\text { transformationnel }\end{array}$ & $\begin{array}{l}\text { Pour un nouveau projet } \\
\text { de société }\end{array}$ & $\begin{array}{l}\text { Tout le système } \\
\text { et ses environnements }\end{array}$ \\
\hline
\end{tabular}

\section{Premier type de réforme : additif/quantitatif}

Le premier type de réforme vise l'élargissement et l'équité (entendue comme parité quantitative) de l'accès à l'éducation par l'accroissement des intrants matériels et humains, particulièrement la construction et l'équipement de nouvelles classes, l'inscription des enfants à l'école et le recrutement de nouveaux enseignants. Ces réformes peuvent également chercher à élever le niveau de scolarisation en augmentant le nombre d'années de l'obligation scolaire avec le même levier. Il est dit additif/quantitatif en ce qu'il se focalise sur le "plus de moyens " pour étendre le modèle en place sinon à tous du moins au plus grand nombre. Ces réformes, qui conservent le même modèle éducatif, ont produit une véritable explosion des effectifs scolaires, explosion présentée comme une démocratisation de l'éducation ou, plus négativement, comme une massification de l'enseignement. En tout cas, elles ont permis à plusieurs dizaines de millions d'enfants d'accéder à l'école. Entrent dans ce type la plupart des politiques menées dans les années 1990 pour atteindre les objectifs de Jomtien, mais aussi bien avant ( $\mathrm{y}$ compris dans les pays développés et 
émergents) et bien après dans les années 2000, avec les mêmes objectifs quantitatifs : politiques d'obligation et de gratuité scolaire, campagnes de scolarisation des filles, écoles rurales communautaires, carte scolaire de proximité, classes à double vacation, classes multigrades, recrutement d'enseignants à coût réduit (moniteurs d'enseignement, maîtres vacataires, volontaires, contractuels...), voire actuellement enseignement à distance, notamment dans l'enseignement supérieur. Ces quatre dernières réformes, tout en s'inscrivant dans la logique additive/quantitative, s'orientent vers la recherche de meilleures formules coût-efficacité dans l'utilisation des moyens afin de «faire plus avec moins », pour combler l'écart entre l'immensité des besoins et la limite des ressources mobilisables.

\section{Deuxième type de réforme : correctif/adaptatif}

Le deuxième type de réforme s'oriente vers l'amélioration de la qualité de l'éducation posée en termes de meilleurs résultats d'apprentissage ou de pertinence renforcée et d'efficacité externe accrue. Il s'attache à diagnostiquer ce qui ne marche pas ou ce qui manque, et à y remédier en s'inspirant le plus souvent de ce qui marche. Il se met dans l'air du temps en adoptant dans la même perspective les innovations curriculaires, pédagogiques, technologiques et autres censées accroître la performance qualitative des systèmes. Ce type de réforme est correctif/adaptatif dans le sens où il ne remet pas en cause le système en place et cherche à corriger ses imperfections et/ ou à l'accommoder aux évolutions scientifiques, techniques et éducatives. Il se satisfait d'ajustements, de remédiations et de mises à jour dans la recherche d'une meilleure qualité de l'éducation. Le levier principal d'action pour le changement tourne autour des intrants intellectuels (curriculum, formation des enseignants et préparation des enfants à l'école) et des processus/procédures d'enseignement-apprentissage et d'organisation de l'école et de la classe. Si l'on s'en tient aux évaluations internationales successives, ces réformes ont permis des avancées notables en direction de l'amélioration des résultats d'apprentissage dans certains pays, notamment en Asie. Il est vrai aussi qu'il a existé aussi des tentatives d'améliorer la qualité de l'éducation par l'accroissement des intrants matériels et du financement (réduction du ratio élèves/ classe dans plusieurs pays ; entre 1970 et 2005 les dépenses publiques en éducation aux États-Unis ont augmenté de $73 \%$ en dollars constants). Mais ces mesures, à elles seules, se sont avérées à chaque fois sans impact significatif sur les résultats d'apprentissage. Dans le type correctif/adaptatif sont classées les réformes curriculaires et pédagogiques [pédagogie par objectifs (PPO), approche par les compétences (APC), approche par les situations (APS), etc.], de nouvelles formules de développement professionnel des enseignants pour accroître leurs performances, l'intégration des technologies de l'information et de la communication (TIC) dans les processus d'enseignement/apprentissage et de nouvelles dimensions éducatives dans le curriculum (paix, environnement, travail productif, entreprenariat, etc.), les innovations dans la gouvernance de l'école et de la classe, des relations nouvelles d'interaction avec l'environnement communautaire, l'enseignement bilingue dans l'enseignement fondamental en Afrique, la « escuela nueva» en Amérique latine, etc. 


\section{Troisième type de réforme : refondateur/transformationnel}

Le troisième type de réforme, contrairement aux deux précédents, ne conserve pas le système en place en l'élargissant et ne se contente pas non plus de l'ajuster. Il le remet en cause pour engager la construction d'un nouveau système intégré à une vision du futur. Dans cette perspective, la réforme redéfinit les finalités de l'éducation et, à partir de là, réoriente et rebâtit tout le système dans ses différentes composantes tout comme ses environnements. Le levier d'action pour le changement est alors constitué de l'ensemble du système, voire de l'ensemble des niveaux du tout social, car il s'agit, à travers l'éducation, de faire émerger un nouveau type d'homme capable de porter un nouveau projet social ou modèle de développement. En fait, la réforme est mise au service d'une rupture avec le système social en place et fait de l'éducation un instrument de la transformation révolutionnaire visée. Dans de telles réformes se retrouvent de véritables « projets-croisades » :

- la « révolution scolaire » de l'entre-deux guerres (1918-1939 en Europe) portée par le "mouvement de l'éducation nouvelle ", qui voulait rompre avec l'école accusée de produire des militaires pour le conflit et construire une nouvelle école, afin de bannir à jamais la guerre en formant des personnes profondément acquises à la paix ;

- l'« école unique du travail » en Union soviétique chargée, après la révolution bolchévique de 1917, de rompre avec l'école tsariste, l'élitisme et la formation de la hiérarchie pour promouvoir une éducation démocratique, gratuite et mixte, orientée vers la formation du nouvel homme prolétarien afin de construire la société communiste ;

- la réforme similaire de l'éducation entamée par la révolution culturelle prolétarienne (1966-1976) déclenchée en Chine par Mao pour l'affirmation de l'hégémonie de la classe ouvrière sur l'éducation, notamment par l'intégration enseignement/ travail productif ;

- l' « école africaine » (expériences de réforme en Guinée, au Ghana, en Tanzanie...) censée se construire à travers une refondation de l'éducation toujours à l'ordre du jour en Afrique, et qui doit renverser le caractère extraverti de l'école héritée de la colonisation dans la perspective de la renaissance culturelle africaine et du développement endogène.

L'éclatement de la Deuxième Guerre mondiale en 1939, la chute du mur de Berlin en 1989, les manifestations de Tian'anmen du mouvement des intellectuels, étudiants et ouvriers chinois de 1989 et l'hégémonie persistante du modèle d'école coloniale en Afrique indiquent que la réussite de ce type de réforme reste une quête.

$\mathrm{Au}$ final, on constate que plus les ambitions de réforme sont élevées, plus l'écart se creuse entre attentes et résultats. Autrement dit, la réussite quantitative semble plus atteignable que la réussite qualitative. C'est ce que montre actuellement l'essor des systèmes éducatifs africains qui ont augmenté les effectifs scolarisés à un rythme accéléré, en particulier entre 2000 et 2015, mais qui peinent à avoir plus d'un élève sur deux en fin de primaire atteignant le seuil minimal dans les apprentissages fondamentaux : lecture et mathématiques. On observe alors que l'amélioration de la qualité est indispensable pour maintenir les acquis de la réussite qualitative. La 
réussite scolaire est aussi plus atteignable que la réussite éducative. Les pays d'Asie qui se sont hissés à la tête des classements PISA en font la démonstration, avec le mal-vivre des jeunes, notamment en Corée du Sud, mettant ainsi en exergue l'importance de combiner à l'acquisition des connaissances et des compétences le développement des valeurs, attitudes et comportements d'épanouissement personnel et collectif pour donner un sens humain à la réforme.

La réflexion sur les facteurs critiques de réussite ou d'échec des réformes permet d'identifier les défis majeurs auxquels est confrontée la conduite du changement en éducation.

\section{CONDUITE DU CHANGEMENT : PRINCIPAUX DÉFIS DE LA RÉUSSITE}

Le premier défi posé à la réforme éducative est de disposer du temps nécessaire au changement. Le «big bang " peut être relativement réussi dans les réformes de type additif/quantitatif. Il est beaucoup plus difficile dans le type correctif/adaptatif et quasi-impossible dans le type refondateur/transformationnel. C'est dire que la réussite de la réforme exige la continuité du changement dans la durée. Dans les régimes politiques modernes, la force des clivages, les alternances démocratiques et diverses résistances sociales et politiques s'opposent à cette continuité dans la durée. Il s'y déroule une succession de réformes parfois contradictoires, sans qu'aucune n'ait le temps de produire l'impact attendu. Elles ont alors plutôt des effets pervers (Tedesco,1994). De ce point de vue, on a pu penser que les pays où a régné un pouvoir autoritaire, en Asie par exemple, ont bénéficié de conditions plus favorables de continuité pour le succès des réformes. En vérité se pose ici la problématique de l'acceptabilité politique et sociale des réformes, qui conditionne leur durabilité. Elle se résout par le dialogue démocratique et la recherche de consensus sur le long terme, comme le montrent des exemples venant de pays d'Europe du Nord.

Le temps contraint aussi le moment des réformes. L'opportunité et la pertinence d'une réforme dépendent de l'étape de développement du système à réformer. Par exemple concernant l'amélioration de la qualité, des chercheurs (Mourshed et al., 2010) ont établi une échelle de cinq niveaux de performance des systèmes éducatifs : faible, correct, bon, très bon, excellent. Au-delà d'un tronc commun de fondamentaux, chaque étape requiert une focalisation sur des réformes spécifiques pour passer à l'étape suivante. Par exemple, un système «faible » voulant passer à « correct» concentre la conduite du changement sur les apprentissages de base en langue et mathématiques. De «bon » à « très bon », le levier principal doit être le renforcement des approches pédagogiques et la transmission de ces savoir-faire entre les enseignants. Le défi est donc ici de bien diagnostiquer l'étape de développement du système, puis d'adapter les attentes en lien avec l'étape suivante et de choisir en conséquence le levier d'action pertinent et efficace pour conduire le changement.

Un autre défi de pertinence et d'efficacité est posé par la duplication de la réussite d'une réforme dans un contexte autre que celui où elle a été réalisée. Le partage des bonnes pratiques et des expériences réussies en matière de réforme est largement promu au plan international. Toutefois, la duplication d'une réforme d'un pays à l'autre se heurte au fait que la simple reproduction des leçons apprises sur 
les conditions et facteurs de succès ignore les déterminants contextuels. Or ce sont précisément ces déterminants contextuels qu'il faut savoir recréer dans le contexte d'accueil pour espérer la réussite. Dans un seul et même pays, cette problématique de reproductibilité peut se poser lorsque l'on veut passer d'un projet-pilote ou d'une expérimentation de la réforme vers la généralisation. Elle nous indique que le défi de la mise à l'échelle d'une réforme n'est pas simplement quantitatif.

Le défi des capacités de réforme soulève la question de la préparation et de l'équipement des institutions et des ressources humaines pour le changement. La conduite du changement requiert de nouvelles capacités de formulation de politique, de gouvernance, de planification, d'élaboration, de simulation financière, de mise en œuvre, d'évaluation et de suivi/ajustement. En l'absence de réponse adéquate au défi des capacités, la faisabilité technique de la réforme peut être mise en doute. Or la réponse habituelle est la formation aux innovations de la réforme. Elle est certes utile mais plus nécessaire encore est le développement de la capacité des différents acteurs d'innover et de trouver des solutions à de nouveaux problèmes avec pour enjeux : l'appropriation active de la réforme, un facteur décisif de succès.

L'effet système constitue pour les réformes un défi majeur souvent ignoré. Le système scolaire en place a son modèle de fonctionnement, ses critères et ses standards de performance, bref sa culture et ses pratiques habituelles. Vouloir changer une ou des composantes de ce système sans se soucier de la convergence avec les autres présente deux risques qui reviennent au même : le rejet ou la dévoration de la substance novatrice de la réforme. Dès lors, lorsque la conduite du changement utilise un ou quelques leviers d'action, elle doit tout au moins avoir une vision systémique du changement permettant, dans le cadre de l'interaction de toutes les composantes du système, d'identifier et de minimiser des effets contradictoires aux options de la réforme.

Dans le même ordre d'idées, le système éducatif lui-même est une composante d'un système social qui l'englobe et lui impose des limites dans le changement. Par exemple, peut-on construire un système scolaire pleinement équitable dans une société inégalitaire ? On peut répondre formellement par l'affirmative en se situant dans la perspective d'avancées significatives que permettent la relative autonomie du système éducatif et sa dynamique propre.

Les chercheurs ont pu mettre en lumière un « effet enseignant » et un " effet école » au sens où, à milieux socio-économiques semblables, des variations s'observent dans la manière d'enseigner et dans la réussite scolaire des élèves de certaines écoles, comme si l'impact du milieu socio-économique avait été neutralisé. (Gauthier et al., 2009).

En fait, le «mur social » fixe toujours des limites qui disent non quand on mesure le poids considérable de l'origine socio-économique dans les déterminants de la scolarité et de la performance des élèves, poids qui peut être certes atténué par des mesures éducatives de compensation mais pas annihilé.

C'est la problématique à laquelle s'attaque le Programme 2030 de l'ONU : transformer le monde dans la perspective du développement durable suppose une approche holistique et interactive de la transformation aux différents niveaux du tout social. L'éducation est donc à traiter à la fois comme objet et comme moteur de transformation en tant que composante des interactions du tout, l'ODD4 étant en 
relation avec les seize autres ODD. Autrement dit, l'éducation subit la transformation du tout social et contribue à celle-ci en interaction avec les autres dimensions. La convergence des différentes dynamiques représente une condition essentielle de succès. Un nouveau défi surgit ici : la nécessité de poser les réformes éducatives dans des approches et projets intersectoriels intégrés au lieu de les considérer dans l'insularité du secteur de l'éducation. C'est ce que souligne le Rapport mondial sur l'éducation de 2016 :

À l'ère des ODD, les approches sectorielles sont inadaptées aux défis transversaux et interdépendants du développement durable (Le Blanc, 2015). Le contexte est en revanche plus propice aux concepts tels que "l'impact collectif » (Kania et Kramer, 2011), qui implique une coordination voulue et structurelle des efforts afin d'atteindre des résultats plus étendus...

Qu'en est-il d'autres défis majeurs, tels que la mobilisation d'une forte volonté politique, un soutien et une participation larges de l'administration et de la société, une conduite mixte du changement alliant top-down et bottom-up, un financement efficace, etc. ? Et surtout, quelle stratégie pour que toutes les actions de changement donnent du sens à la réussite des réformes en impactant positivement et durablement les apprentissages qui se déroulent en classe ? Est ici impliqué le défi le plus critique des réformes : le changement de cultures et de pratiques des enseignants en direction de l'innovation. Au-delà de la formation et d'une simple approche d'apports externes pour le renforcement de capacités, il faut tourner le regard vers l'approfondissement et l'opérationnalisation du concept de développement professionnel basé sur des pratiques réflexives d'auto et d'inter-évaluation/apprentissage.

Au bilan, la multiplicité et la complexité de tous ces défis posés à la conduite du changement ne font-ils pas de la réussite attendue des réformes une gageure ? Elles suscitent tout au moins un scepticisme grandissant sur la capacité des réformes éducatives à répondre aux attentes de changement des systèmes.

À la réflexion, la plupart des déceptions s'expliquent par ceci : ce qui est attendu des réformes s'assimile le plus souvent à un changement révolutionnaire. Cette espérance est aussi alimentée par les promoteurs des réformes à travers des discours manichéens où ce qui est à réformer est radicalement remis en cause comme mal absolu et ce qui est nouveau magnifié de manière idyllique (Gauthier et al., 2009). Or la réforme n'est pas une révolution tant du point de vue du temps de sa réalisation que de celui de la nature et du rythme du changement qu'elle doit opérer.

\section{UNE CONDUITE DU CHANGEMENT EN ÉDUCATION ORIENTÉE VERS LE DÉVELOPPEMENT SPIRALAIRE}

Il faut donc revenir au sens profond mais plus modeste du mot réforme pour ne pas en attendre un renversement/rupture de type révolutionnaire. Il s'agit plutôt de la concevoir et de la conduire en tant que processus continu et progressif d'amélioration de la performance d'un système éducatif. Certains parlent de "réformisme permanent ». L'approche s'appuie sur les leçons à tirer des défis identifiés ci-dessus. 
Le premier pas dans cette direction consiste à bâtir un consensus national de long terme sur les objectifs et options stratégiques de changement en relation avec une vision partagée du futur. Le succès exige un dialogue démocratique authentique et ouvert à toutes les parties prenantes du système et aux différentes composantes de la société. Il est essentiel, pour la durabilité, que le résultat obtenu réponde à une demande profonde de la société et non à une simple offre gouvernementale ou, encore moins, à des positions partisanes. Un tel consensus national peut transcender les oppositions classiques et les alternances démocratiques.

Une fois l'orientation consensuelle définie et située sur le long terme intervient la planification du changement. L'approche doit être :

- intersectorielle au regard des interactions avec d'autres secteurs pour identifier les partenariats possibles, développer des projets intégrés de changement, rechercher " l'impact collectif » et éviter ainsi de concevoir et de mettre en œuvre le changement en éducation dans l'insularité ;

- systémique, au double sens de la prise en compte de toutes les composantes du système et des différents sous-secteurs du secteur éducation, afin de considérer les interactions à l'intérieur du système et d'orienter la dynamique d'ensemble en direction des objectifs de la réforme ;

- centrale et décentralisée, à travers l'élaboration de plans d'actions du niveau macro ou national aux niveaux méso (intermédiaire décentralisé et/ou déconcentré) et micro (établissement et classe).

L'approche participative doit ouvrir des espaces d'initiative et de création pour les acteurs à tous ces niveaux, tout en renforçant leur capacité d'innovation. Dans cette perspective, l'apprentissage par l'auto-évaluation et l'inter-évaluation des performances, la recherche collégiale et l'échange entre pairs de solutions aux problèmes rencontrés, le renforcement de la déontologie et de l'engagement professionnels à travers la promotion des activités associatives de la profession s'avèrent très productifs, notamment pour les acteurs clés du changement que sont les enseignants. En effet, c'est grâce à cet apprentissage et à ce développement professionnels continus que les enseignants et les autres acteurs de la réforme peuvent répondre aux exigences de capacité de changement et participer activement au développement de l'innovation.

Tout en adoptant ainsi des approches qui permettent de relever les défis majeurs analysés ci-dessus, la conduite du changement a besoin d'un allié critique pour le développement spiralaire : la recherche. C'est grâce à cette dernière qu'elle peut opérer les choix opérationnels les plus efficaces, réussir l'évaluation diagnostique et prospective à chaque étape de développement, en tirer les enseignements utiles pour améliorer l'étape suivante tout en anticipant les évolutions à intégrer. Par exemple, quelles options de gouvernance, curriculaires, pédagogiques, organisationnelles marchent le mieux compte tenu du contexte national, de l'état du système et de l'orientation stratégique choisie pour le changement ? À l'issue d'une étape du processus de changement, quelles leçons tirées peuvent permettre d'améliorer l'étape suivante ? Comment mettre en œuvre ces leçons le plus efficacement possible ? Quelles sont les tendances d'évolution du changement à anticiper ? S'en tenir ici aux opinions n'ayant aucune base scientifique fait errer la conduite du changement dans nombre de réformes. C'est pourquoi la connaissance et l'utilisation pertinente des 
résultats de la recherche sont ici capitales pour répondre adéquatement à de telles questions. Elles permettent notamment à la conduite du changement d'apprendre de l'expérience écoulée et des découvertes scientifiques pour l'amélioration continue de l'action prochaine et pour des sauts qualitatifs à chaque étape. C'est dans cette perspective que s'inscrit le développement spiralaire du changement qui permet à un système de perfectionner sans cesse ses performances par bonds successifs.

Ce développement spiralaire doit s'ouvrir aujourd'hui sur les tendances d'évolution des systèmes éducatifs dans la perspective du développement durable. En effet, un glissement du concept de durabilité s'est opéré de l'environnemental à l'économique, au social et au culturel. En contre-champ de cette compréhension élargie du concept de développement durable se profile également une autre dimension : celle liée à la durabilité des acquis de l'apprentissage. Elle interpelle fortement dans un "village planétaire » où la révolution médiatique, technologique et scientifique devenue quasi-permanente produit des changements et des mutations si rapides qu'ils rendent vite obsolètes les connaissances et les compétences acquises. L'interpellation s'avère plus pressante avec l'hégémonie de plus en plus accentuée des sociétés et des économies du savoir et de l'innovation. Il se trouve que la durabilité de l'apprentissage soulève au moins deux problématiques transversales : celle de l'apprentissage tout au long de la vie, devenu une nécessité ,et celle de l'approche intersectorielle de l'éducation, qui appelle des projets intersectoriels intégrés. Ces deux problématiques remettent en question le monopole académique sur l'acquisition du savoir. Elles posent à la conduite du changement en éducation l'exigence 54 de construire de nouvelles sociétés et communautés apprenantes ou, autrement dit, d'organiser l'intelligence coopérative de la société pour des possibilités d'apprentissage en tout lieu et à tout âge (ODD4). Il s'agit de mobiliser et d'offrir une gamme diversifiée d'opportunités d'apprentissage adaptées à la diversité des besoins et situations, donc à la demande. L'apprentissage tout au long de la vie englobe toutes les activités d'apprentissage entreprises au cours de l'existence dans le but d'améliorer ses connaissances, compétences et aptitudes à des fins personnelles, civiques, sociales ou professionnelles. Il intègre l'éducation au sein de la famille et de la communauté, l'apprentissage dans le travail, les lieux de loisirs et dans la vie de tous les jours ainsi que les connaissances, les valeurs et les comportements acquis de manière intergénérationnelle, entre pairs, par l'auto-apprentissage et l'expérience, à distance ou à travers les associations, les organisations religieuses, les syndicats, les médias, les réseaux sociaux... Tous les niveaux du tout social y participent : le politique, l'économique, le social, le culturel... L'école du futur résidera donc en tout lieu et sera ouverte à tous les âges. Elle se déploiera à travers des communautés apprenantes formelles, non-formelles et informelles ainsi que par tous les types d'apprentissage. Le système scolaire et universitaire, bref académique, l'intégrera s'il se donne la capacité de tisser des partenariats éducatifs appropriés avec tous les secteurs identifiés comme pertinents et qui concourent à un apprentissage intra et extra muros et à une éducation ouverte à la demande ou à l'initiative de la société.

Conduire le changement en éducation vers ce futur déjà à l'œuvre dans le présent constitue un nouveau défi. Il pose le dépassement des trois types de réforme analysés soit parce que leurs choix stratégiques sont trop étroits pour prendre en considération l'exigence systémique et intersectorielle, soit parce que la conception 
du changement à conduire est hors de portée. En les dépassant, le développement spiralaire du changement en éducation les intègre à la fois dans leurs attentes et par l'approche systémique. C'est une réforme permanente qui a en vue tous les leviers et les actionne en fonction des éclairages tirés des évaluations périodiques et des résultats de la recherche tout en donnant un sens au mouvement dans la direction stratégique prise vers la demande consensuelle de la société. Une fois adopté et mis en œuvre, il intègre les fondamentaux des systèmes éducatifs tout comme les stratégies spécifiques liées aux cinq étapes définies plus haut. Il n'est jamais achevé car son horizon d'amélioration des performances du système éducatif est infini. Évidemment chaque pays l'adopte selon des modalités et rythmes de réalisation liés aux possibilités qu'offre son contexte de développement et sa vision du futur. Mais pour tous, la stratégie commune est assimilable à une révolution silencieuse permanente.

\section{RÉFÉRENCES BIBLIOGRAPHIQUES}

LE BLANC D. (2015). Towards integration at last? The sustainable development goals as a network of targets, DESA Working Paper No. 141 ST/ESA/2015/DWP/141. En ligne] https://www.un.org/esa/desa/papers/2015/wp141_2015.pdf

GAUTHIER C., BISSONNETTE S. et RICHARD M. (2009). « Réussite scolaire et réformes éducatives ». Revue de recherche appliquée sur l'apprentissage, $\mathrm{n}^{\circ} 2$ (numéro spécial), article 1 , p. 1-15.

KANIA J. et KRAMER M. (2011). "Collective impact ». Stanford Social Review Innovation Review. [En ligne] https://ssir.org/articles/entry/collective_impact\#

MOURSHED M., CHIJIOKE C. et BARBER M. (2010). How the world's most improved school systems keep getting better, McKinsey\&Company.

TEDESCO J.C. (1994). "Tendances actuelles des réformes éducatives ». Revue internationale d'éducation de Sèvres, $\mathrm{n}^{\circ}$ 1, p. 77-85. [En ligne] http://journals.openedition.org/ ries/4307; DOI : 10.4000/ries.4307 